\title{
Multilinguales
}

1 | 2013

Pratiques littéraires, linguistiques, pédagogiques,

didactiques et médiations culturelles contemporaines

\section{La photographie de guerre dans le démantèlement de Rachid Boudjedra : Objet culturel/objet textuel}

The War Photography of "The Dismantling" (Le Démantèlement) of Rachid Boudjedra: Cultural Object/Textual Object

\section{Mounya Belhocine}

\section{OpenEdition}

\section{Journals}

Édition électronique

URL : https://journals.openedition.org/multilinguales/2863

DOI : $10.4000 /$ multilinguales.2863

ISSN : 2335-1853

\section{Éditeur}

Université Abderrahmane Mira - Bejaia

\section{Édition imprimée}

Date de publication : 1 juin 2013

Pagination : 19-29

ISSN : 2335-1535

Référence électronique

Mounya Belhocine, «La photographie de guerre dans le démantèlement de Rachid Boudjedra : Objet culturel/objet textuel », Multilinguales [En ligne], 1 | 2013, mis en ligne le 01 juin 2013, consulté le 02 juillet 2021. URL : http://journals.openedition.org/multilinguales/2863 ; DOI : https://doi.org/10.4000/ multilinguales.2863

Ce document a été généré automatiquement le 2 juillet 2021.

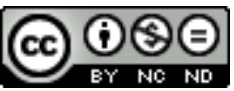

Multilinguales est mise à disposition selon les termes de la Licence Creative Commons Attribution Pas d'Utilisation Commerciale - Pas de Modification 4.0 International 


\title{
La photographie de guerre dans le démantèlement de Rachid Boudjedra : Objet culturel/objet textuel
}

\author{
The War Photography of "The Dismantling" (Le Démantèlement)of Rachid \\ Boudjedra: Cultural Object/Textual Object
}

Mounya Belhocine

1 Les objets de roman suscitent l'intérêt des critiques depuis de nombreuses années, qu'il s'agisse de ceux des romans réalistes ${ }^{1}$, ou de ceux du nouveau roman ${ }^{2}$. Leurs investigations sont, pour la plupart, soit d'ordre psychanalytique, soit sociologique. Mais l'objet auquel nous nous intéressons ici, la photographie dans Le Démantèlement de Rachid Boudjedra (Denoël, 1982), appelle, de notre point de vue, un troisième type de lecture dont nous n'avons pour l'heure que peu d'exemples, la lecture ethnocritique, parce que c'est un objet textuel fortement marqué au niveau culturel.

Cette étude nous a été inspirée par l'approche des Misérables de Victor Hugo proposée par l'ethnocriticien Guillaume Drouet et, en particulier, l'étude qu'il consacre au «bâton» de Jean Valjean, dans un article intitulé « Le bâton de Jean Valjean. Marcher, sculpter, raconter » (Poétique $\mathrm{N}^{\circ}$ 140, $2004:$ 467-482) 2.

Il commence par justifier la focalisation sur cet objet en ce qu'elle est dictée explicitement par le texte hugolien: "en effet, "un détail" dans la caractérisation du personnage principal a attiré notre attention: tout au long du roman, Jean Valjean s'arme de "bâtons" » (Drouet, 2004 : 267).

Ensuite, Guillaume Drouet va s'appliquer à mettre en valeur la dimension symbolique de cet objet en établissant les liens qu'il tisse, dans le texte, avec «les pratiques » de "la culture pastorale", en se proposant de "traiter le bâton (...) comme un instrument dont la symbolique s'ancre dans des pratiques spécifiques, quotidiennes, rituelles ou artistiques, que l'œuvre retextualise et retravaille dans des directions particulières " (Drouet, 2004 : 478).

5 Enfin, après avoir cerné les «dimensions particulières" de ce «bâton», il conclut son étude en ces termes : 
En fait, il semble qu'en abolissant les distinctions des pratiques Hugo ait perçu, à travers son bâton, la dimension anthropologique du symbolique, véritable processus de "domestication" aboutissant à la "création [...] d'un espace et d'un temps maîtrisables " (...). (Drouet, $2004: 479)$

6 Cette lecture du «bâton de Valjean, qui aboutit à la dimension anthropologique du symbolique du processus de création ", met au jour la culture du texte, une culture qu'il n'invente pas, mais qu'il réinvente et d'où il tire en grande partie son effet. La médiation assurée par la photographie, dans le roman de Rachid Boudjedra, débouche sur le même résultat : elle est « un instrument symbolique » à travers lequel est donnée à lire la tentative de réécrire un certain épisode de l'histoire de l'Algérie.

Deux raisons majeures ont déterminé notre choix de lecture ethnocritique de la "photographie de Tahar El Ghomri »:

- la première est relative aux romans de Rachid Boudjedra qui, à l'instar du roman hugolien, ou des romans algériens et maghrébins en général, sont fortement marqués culturellement ;

- la seconde est inhérente à l'objet en question, une photographie de guerre, propriété du personnage principal et qui occupe une place stratégique dans le récit.

Elle apparaît dès les premières lignes de l'incipit du Démantèlement : "Il ne portait même pas de carte d'identité ni aucun papier sur lui (...), à l'exception de la photographie (...) - la photographie unique dont il portait le seul original existant » (7).

Le personnage principal tire son identité de cette photographie en lieu et place des « papiers » d'« identité ». Il est «l'homme à la photographie » (8).

Elle est également présente à l'excipit, dans ses dernières lignes : "Il n'avait rien dit de son enfance et de sa vie personnelle mais avait passé son temps à l'écrire, la ressasser et la décrire, à porter la vieille photographie qui n'en pouvait plus » (307). L'acte d'« écrire (sa vie), (de) la ressasser et (de) la décrire " se superpose à celui de " porter la vieille photographie ", pour induire leur équivalence et leur similitude : raconter son histoire et donc l'histoire - les deux sont intimement liées - équivaut à «porter la photographie ». L'histoire et la photographie sont associées dans le roman au point de se confondre.

11 Dans l'espace qui sépare l'incipit de l'excipit, l'évocation de cette photographie essaime le texte, générant les récits des personnages qui sont assis au premier rang, et celui de la photographie elle-même. Celle-ci est narrativisée puisqu'elle subit l'usure du temps au fur et à mesure que le récit avance, et qu'elle évolue dans l'espace : elle passe de Tahar El Ghomri (en qui elle se fondait) au mur de la pièce sur lequel elle est épinglée (juste « en face » de lui).

12 Nous pensons qu'en explorant un tel objet culturel, la "photographie » de guerre de Tahar El Ghomri, nous parviendrons à investir la «dimension anthropologique du symbolique » du roman de Rachid Boudjedra. Rappelons que pour G. Drouet, les objets culturels sont des « points nodaux de la culture, puisqu'ils participent à ses trois dimensions "fonctionnelle, mythique, esthétique"4 - [et qu'] ils ne peuvent se comprendre que comme carrefours de pratiques, médiateurs grâce auxquels les hommes ont prise sur leur environnement ». L'environnement dont il s'agit ici est celui de la guerre de libération de l'Algérie et de son écriture après l'indépendance.

13 A l'instar de Drouet, nous allons traiter la photographie, qui est un élément de cet environnement, dans ses trois dimensions : fonctionnelle, mythique et esthétique. 


\section{La photographie : une archive de la guerre}

Dans le texte de Rachid Boudjedra, la photographie de guerre, en tant qu'objet culturel, ne s'éloigne pas de sa fonction référentielle initiale, c'est-à-dire attester de la véracité des faits :

Il n'ignorait pas que la destruction de ce document (la photographie) serait un désastre parce que la photographie représentait beaucoup plus qu'aucun papier officiel colorié de tampons de toutes les couleurs et enjolivé avec un tas de timbres multicolores. Elle était l'unique preuve tangible de ce qui s'était passé réellement. (18)

La photographie vaut un témoignage officiel, et celui-ci est d'autant plus précieux qu'il est à la fois unique du point de vue de son contenu et fragile du point de vue de son support. A cette valeur de l'objet en tant qu'archive de guerre, s'ajoute celle en tant qu'objet narratif associé à la quête d'un maquisard qui tente d'accomplir un devoir de mémoire.

En effet, le projet d'écriture du roman est une interrogation explicite sur le sens de l'Histoire: «qu'est-ce que l'histoire?» est une question qui parcourt tout le roman. Elle est portée par deux personnages qui ne sont pas satisfaits du discours sur la guerre d'Algérie : la jeune bibliothécaire, Selma, qui réfute la version officielle de certains événements historiques, et le vieux combattant communiste, Tahar El Ghomri, qui tente de lever le voile sur certains épisodes de l'Histoire de l'Algérie. Selma exige (vainement) d'El Ghomri le rétablissement de la vérité des faits historiques ; lui, acculé par son interlocutrice, s'accroche à la photographie prise avec ses amis au maquis, vingt-cinq ans plus tôt, en décembre 1956. Celle-ci est décrite, à maints endroits du texte, comme une archive de la guerre :

Elle est bistre. Son papier est ancien et grenu, fendillé à quelques endroits de telle sorte que certains des visages représentés sur le cliché donnent l'impression qu'ils sont coupés ou plutôt lacérés (...) au niveau des joues, du front et du menton. Elle retourne la photographie et lit sur le dos Dec. 56. (...) La photo représente au premier rang un groupe de cinq personnes assises à même le sol, habillées de kachabias et les têtes enroulées de chèches, tenant entre les mains de vieilles armes quelque peu ridicules et saugrenues. (54-55)

Les occurrences renvoyant à la date, à la tenue vestimentaire et aux armes inscrivent la photographie comme "photographie de guerre", ou plus précisément comme "photographie du maquis", puisqu'il s'agit de "maquisards» pendant la guerre d'Algérie : « il se remettait à parler de la photographie et de ceux qui y figuraient, du maquis où elle a été prise, du type d'armes qu'ils possédaient, à l'époque, et d'autres détails » (130).

Cette description nous permet d'évaluer la charge culturelle insufflée à cette photographie. Les kachabias (sorte de burnous) et les chèches (sorte de turban) campent le type du maquisard algérien véhiculé par les archives visuelles sur la guerre d'Algérie. Cette conformité avec l'objet culturel est confortée par la pause des maquisards d'après les photos d'archives : ceux du premier rang assis, souvent à même le sol, et les autres debout.

19 Cette photographie de guerre appartient donc au réel. C'est une photographie de guerre qui est textuellement une " archive de guerre »:

Nous avions un ennemi commun et la même cause. Nous sûmes que la révolution, c'était l'enfer arrosé de sang et de vomi. Nos entrailles explosaient entre nos mains et bleuissaient sous le dard des mouches espiègles. Les journaux s'étaient moqués 
de nous au début (...). Mais ceux qui s'étaient moqués de nous, la veille, avaient changé brusquement d'avis : ils se mirent à décréter, sous l'effet de la panique, n'importe quoi: tribunaux militaires, renforts en hommes et en armes, condamnations à mort, couvre-feu, état de siège ... (189-190) étranger » (192), "après un siècle et demi de silence, de peur, d'obséquiosité» (188). Ce « nous » désigne les communistes qui ont fait alliance avec les autres combattants, en référence à un fait historique avéré: en 1956, a été négociée l'intégration des combattants communistes du Parti Communiste Algérien (PCA) au sein de l'Armée de Libération Nationale (ALN).

21 Ainsi, ceux de la photographie sont, pour Tahar El Ghomri, «des camarades. Des communistes» (114) et "les cinq hommes du premier rang étaient tous membres du comité central » (147). Ils avaient combattu ensemble: «il s'était réfugié sur la crête du piton inexpugnable pour harceler l'ennemi et sauver sa peau, en compagnie de ses camarades (...) et les autres qui apparaissaient sur la photo » (123). égorgés...égorgés! Par leurs frères de combat... » (89). Le roman évoque ici de façon explicite un épisode de la guerre d'Algérie textuellement identifié comme "la chasse aux rouges» (151):

Nous aussi nous avons combattu, pris le maquis, détourné la plus grande quantité d'armes que la France ait jamais perdue, fabriqué des bombes, organisé nos propres groupes de choc, créé des réseaux étanches dans les villes, donné à la résistance des hommes de toutes confessions : musulmans, juifs, chrétiens, athées, zorostroades ! Envoyé à la guillotine des camarades (...). Mais qui en parle ? Pourquoi ce rempart de silence autour de notre contribution? (184)

C'est précisément ce «silence» que Selma veut rompre en harcelant de questions Tahar El Ghomri, seul survivant parmi les combattants communistes de la photographie: "elle est maintenant obsédée par le désir de connaître avec précision et certitude quel a été le rôle joué par le Parti durant la guerre anticoloniale » (202-203). Et c'est dans le but de combler ce même « silence » que Tahar El Ghomri s'acharne à écrire :

Tahar El Ghomri n'avait pas d'autre préoccupation que l'écriture de l'histoire (...). Puis à nouveau il revenait à l'écriture de l'histoire, telle qu'il l'avait vécue à travers ses luttes politiques, son engagement actif dans la guerre, ses règlements de comptes internes. (199-200)

Son objectif est de « clarifier l'histoire de son pays et surtout l'histoire de cette période cruciale de la guerre, soulever la chape du silence qui s'était abattue sur le rôle joué par ses camarades ; enlever la poussière sur l'engagement d'un groupe qui en avait tant vu et tant bavé (...) » (297).

Dans cette entreprise de l'écriture de l'histoire, la photographie joue le rôle de médiateur entre le passé et le présent: "Tahar El Ghomri se rappelait alors la photographie. Il la prenait avec douceur. Il pensait qu'elle était plus précieuse et plus éloquente que n'importe quelle carte d'identité ou que n'importe quel monument du souvenir » (96).

La photographie de guerre de Tahar El Ghomri est, par conséquent, un signe identitaire et un symbole du souvenir. Elle signifie au second degré. 


\section{La photographie : un objet (esthétique) textuel}

27 La photographie, être de papier, déroule le temps du récit dans le roman de Rachid Boudjedra. Elle déclenche l'intrigue, rompt le silence du personnage, et fait avancer le récit en révélant l'identité et l'histoire de chacun des compagnons d'armes de Tahar El Ghomri. La photographie, comme document historique, devient dans le texte de Boudjedra "un objet de la narration». Décrite de façon détaillée dans quelques passages ressassés tout au long du texte, elle détermine le rythme du récit et constitue sa pierre angulaire.

Cet objet nous permet également de dresser un paradigme des différentes identifications qui le confortent dans son statut d'objet culturel (et cultuel) dans le texte de Rachid Boudjedra :

Quant à la photographie! Tu voudrais éliminer l'espace et figer le temps... Tu ne possèdes ni calendrier, ni emploi du temps, ni almanach (...). N'est-ce pas une façon de fuir le temps? (...) Tu gardes jalousement le secret de tes camarades représentés sur cette maudite photographie que tu portes sur toi comme une amulette magique, un fétiche de bonne femme, un porte-bonheur en celluloï... Mais qui es-tu donc? Et eux, qui sont-ils? (34-35)

Nous constatons que la description de la photographie révèle sa surdétermination culturelle. Elle est assimilée à un fétiche aux vertus protectrices et bienfaitrices. Cependant, son propriétaire est parfois persécuté et harcelé par sa seule présence qui devient oppressante : « la photographie bistre avait perdu ses couleurs; et il ne lui restait rien d'autre que ce trophée minable... (14). Tahar El Ghomri entretient avec cet objet une relation contrastée, à la fois de rejet et d'attirance parce qu'il lui renvoie tout le poids de sa responsabilité et de sa culpabilité vis-à-vis de ses compagnons d'armes et de l'Histoire :

En fait, il s'était suicidé. Il ne s'était pas soigné depuis l'année où le docteur fut égorgé ; et il avait brûlé ses poumons au petit feu de la culpabilité et de la mauvaise conscience. Il ne s'était jamais remis de cette chance qui lui avait permis de s'enfuir et de sauver sa peau; (...). Il regrettait de ne pas s'être rendu à ses propres compagnons d'armes qui voulaient l'égorger. (296)

Longtemps enfouie (comme ses remords) au fond d'une poche de sa veste - Tahar El Ghomri, "feignant d'oublier la photographie ou de ne lui accorder aucune importance " (129) -, celle-ci est enfin épinglée au mur pour lui faire face, comme l'objet de «sa culpabilité et de sa mauvaise conscience ": "il se décida à la coller au mur avec des punaises, parce qu'il avait rompu ses liens avec la vie et compris qu'il lui fallut mourir très vite» (293). En l' « extirpant » de son corps pour la poser devant lui, il a signé sa mort.

31 Cette photographie balise la destinée du personnage et reflète son trajet du début à la fin du roman qui s'achève sur son suicide. L'état de la «vieille» photographie s'est aggravé au contact de la lumière - elle était «habituée à l'obscurité et à la moiteur de sa poche » (265) - en même temps que l'état de santé de son propriétaire quand il a décidé de quitter sa vie de reclus: "La photographie ne cessait pas de se dégrader comme ses poumons ne cessaient pas de se gâter » (274).

32 En plus de véhiculer un savoir culturel qui sert à identifier ce qu'elle représente, la photographie est non seulement le double symbolique du personnage principal, mais aussi le double symbolique de son histoire :

Il avait donc collé la photographie au mur (...) et s'était senti soulagé du poids des années et des morts. (...) Il avait voulu en finir avec le souvenir lancinant de ce 
massacre du 8 mai 1945 et se débarrasser de la blafardise du passé, de ses obsessions décrépites et vieillottes, de ses rancunes verglacées et d'autres sentiments encore, telles ces choses dont l'entêtement répétitif, à satiété, affole, déboussole et désagrège un individu, comme cela avait été le cas de la photographie devenue un complexe qui bloquait ses vaisseaux sanguins et ses viscères, sa vision du monde et sa conception de l'histoire. (273) jalonnant le cycle de la vie, comme ceux que l'ethnologue Arnold Van Gennep appelle « rites de passage ». Dans son ouvrage intitulé Les Rites de passage (1909) ${ }^{5}$, il explique que tout rite comprend trois phases successives : les rites de séparation, les rites de marge et les rites d'agrégation (Van Gennep, cité par Scarpa, 2009 a : 161). Cependant, certains individus ne parviennent pas à passer avec succès ces trois étapes. Ils restent au second stade que Victor Turner désigne comme étant celui de la liminalité ${ }^{\text {: }}$

Le concept de liminalité a été principalement développé par les anthropologues Arnold Van Gennep et Victor Turner afin de définir l'état identitaire des «initiés » qui traversent les rites de passage comme des moments où ils sont dépossédés de tout, où leur statut devient flou et leur être vidé de sa substance afin de les préparer à recevoir leur nouveau soi.?

" moments où ils sont dépossédés de tout, où leur statut devient flou » font d'eux des personnages de l'entre-deux, ou liminaires. Selon Marie Scarpa, le personnage liminaire " n'est définissable ni par son statut antérieur ni par le statut qui l'attend tout comme il prend déjà, à la fois, un peu des traits de chacun de ces états " (Scarpa, 2009 b). Elle précise que

La construction de l'identité se fait dans l'exploration des limites, des frontières (toujours labiles, en fonction des contextes et des moments de la vie, mais toujours aussi culturellement réglées) sur lesquelles se fondent la cosmologie d'un groupe social, d'une communauté : limites entre les vivants et les morts, le masculin et le féminin, le civilisé et le sauvage, etc. (28)

Dans Le Démantèlement, il s'agit précisément des « limites entre les vivants et les morts ", et le personnage Tahar El Ghomri peut être considéré comme un passant. Il a à " passer » de la vie à la mort. Or, il est considéré comme mort parmi les vivants. Mais il n'est pas 
réellement mort pour qu'il puisse enfin rejoindre ses amis morts, même s'il poursuit ce but depuis des années. Va-t-il réussir son passage?

Dans la phase préliminaire, ou phase de séparation, Tahar El Ghomri reste bloqué pendant vingt-cinq ans, tenu en (sur)vie par la photographie. Il est resté cloîtré dans sa "bicoque", refusant tout contact avec le monde extérieur. Il se reproche toujours d'être resté vivant alors que tous ses camarades sont morts plusieurs années auparavant :

[Il] se rappelle de temps à autre la photographie et rougit d'être vivant, alors que tous les autres comparses figurant sur le cliché étaient bel et bien morts, jusqu'au dernier, depuis belle lurette. (...) Revient à la photo. Veut se faufiler dans l'armoire de son corps. Inutilement, car elle est fermée à double tour. Le vieil homme se recroqueville sur lui-même. Fait des gestes de diversion. (...) Revient à la photographie. (...) Ceux qui sont morts sont enterrés. Trop facile. La pièce à conviction lui brûle les doigts et le fascine littéralement. (Boudjedra, 1982 : 29)

Tahar El Ghomri a honte de ne pas être mort et enterré, comme ses amis sur la photographie qui lui rappelle qu'il est encore en vie. Toute cette phase correspond à celle où la photographie ne quittait pas la poche de sa veste parce qu'il était prisonnier de ce moment où le cliché avait été pris, vingt-cinq ans plus tôt, en décembre 1956.

Pour l'étape de marge, Tahar El Ghomri oscille dans un entre-deux de la vie et de la mort. Il accède à cette phase en sortant la photographie de sa poche (après vingt-cinq ans) pour la coller au mur, et en décidant de reprendre contact avec le monde extérieur :

Il avait donc collé la photographie sur le mur à l'aide de punaises et s'était senti soulagé du poids des années et des morts. Même sa chambre miteuse lui semblait plus facile à supporter depuis qu'il l'avait repeinte, restaurée, avait changé ses meubles de place, troué une fenêtre réelle cette fois-ci, nettoyé le sol, redistribué son espace, installé l'électricité comme s'il s'agissait d'une invention récente qui l'avait laissé perplexe...(...) Cependant, il ne pouvait oublier les camarades qui depuis qu'il les avait épinglés au mur avaient retrouvé une certaine vie et s'était mis à l'entourer comme durant les jours où ils étaient réunis dans le même maquis, avant qu'ils soient tués l'un après l'autre. (...) Depuis qu'il avait mis entre lui et la photo la barrière de son mur, la chambre s'était remplie de l'écho de leurs voix mortuaires, de leurs mouvements cadavériques, de leurs démarches ramollies, de leur nomadisation ineffable... (272-275)

Le rapprochement avec les vivants ne lui fait pas oublier les morts, bien au contraire : leur présence se fait plus forte au point que nous pouvons dire que les deux mondes, celui des morts et celui des vivants, cohabitent pendant cette période de marge où le personnage se retrouve à la fois dans l'un et dans l'autre.

Quant à la dernière étape, celle d'agrégation, elle correspond à son retour au sein du groupe des amis morts : le passant va être réintégré au groupe qu'il avait quitté lors de la phase de séparation. Tahar El Ghomri va rejoindre ses amis, en les imitant dans la mort :

Il était donc mort ! Elle l'avait trouvé baignant dans une mare de sang et la bouche pleine de gros caillots, déjà solidifiés, comme s'il avait eu envie d'imiter le docteur Cogniot et l'Allemand égorgés tous les deux dans les râles de l'agonie interminable parce que le couteau rouillé n'arrivait pas à trancher la carotide dure et gélatineuse. Comme s'il avait fait exprès, aussi, de mourir dans une atroce agonie, à la façon de Sidi Ahmed Inal aspergé d'essence et brûlé vif. Comme s'il avait voulu effriter ses poumons à la manière de Bouali Taleb qui avait été déchiqueté par sa propre bombe. (303) 
mble avoir effectué avec succès son rite de passage de la vie à la mort. A-t-il pour autant échappé à la phase liminaire ? Ce rituel de passage ayant été contrarié durant une très longue période, et le personnage s'étant donné lui-même la mort, cette hypothèse est plus que suspecte parce que cela fait de lui un «sur-initié », donc un personnage définitivement liminaire.

Nous nous référons à Marie Scarpa qui envisage la possibilité de distinguer une nouvelle catégorie de personnage liminaire qu'elle propose d'appeler «le non initié surinitié », et qu'elle définit en ces termes :

Dans la mesure où notre personnage liminaire (...) ne parvient pas à revenir de cette altérité ; qu'il est (...) un non initié, un mal initié ou un sur-initié (voire le tout en même temps), il est placé souvent, dans le système des normes culturelles, du côté du pôle le moins positif ou le plus problématique.(...) Parfois, donc, la fiction moderne fait endosser au personnage liminaire, en l'historicisant toujours, en le démythifiant (démystifiant?) souvent, le rôle, anciennement dévolu à certains " héros », d'explorer «la côte mal taillée en quoi consiste le réel », les "vérités négatives " sur lesquelles peuvent se construire les systèmes culturels et de faire surgir, éventuellement, d'autres possibles. (Scarpa, 2009 a : 34-35)

Tahar El Ghomri, qui a vécu dans la clandestinité pendant vingt-cinq ans, accroché à cette photographie et au moment de l'histoire dont elle est la preuve unique, qui s'est finalement suicidé sous le poids du remords, est un personnage liminaire "non initié sur-initié ", à qui a été confié "le rôle d'explorer les vérités négatives sur lesquelles " s'est construit le discours sur « la guerre anticoloniale » de l'Algérie.

Cette sur-initiation est confortée par le fait que ce personnage, même mort, continue de transgresser les interdits des vivants :

Ce fut donc la fin. L'imam refusa de dire la prière des morts sur le cadavre de Tahar El Ghomri ce qui donna le fou rire à Selma devant le comportement fanatique du vieux clerc dévot (...) qu'elle gifla publiquement pour lui cracher sa haine et son mépris. Tahar El Ghomri fut donc enterré solitairement et sans la bénédiction divine. (...) Même le croque-mort s'en mêla et refusa de descendre le cadavre dans le trou si Selma ne s'en allait pas. Elle lui tint tête et Latif régla le problème en graissant largement la patte du bonhomme. (305)

Cette scène fait écho explicitement à celle des funérailles d'un de ses amis, Bouali Taleb, autre personnage de la photographie : les imams avaient refusé de célébrer la prière des morts parce qu'il était communiste et ses amis ont dû enlever l'imam de La Grande Mosquée pour le forcer à procéder au rituel funéraire.

Ainsi, le rituel de passage est escamoté, renforçant la thèse du personnage liminaire non initié sur-initié d'autant que Tahar El Ghomri veut connaître la même mort que ses amis pour pouvoir les rejoindre : « il avait eu une belle mort (...). Il était mort comme il l'avait voulu, entouré de son propre sang et y baignant généreusement: "c'est là l'essentiel! " " (305). Si le personnage est toujours dans l'imitation de ses amis même dans la mort, cela signifie bien que, même mort, il n'est pas "passé ». Pour cela, il aurait fallu qu'il ne mette pas en scène sa propre mort, qu'il n' " arrange " pas son " passage ». Nous en déduisons que le personnage principal est un personnage irrémédiablement liminaire.

50 Nous pouvons conclure en rappelant que la photographie de guerre, objet culturel fortement textualisé, est le double métaphorique du personnage et de son histoire, qui ne se distingue pas de l'Histoire de la guerre de libération de l'Algérie. En tant que tel, elle est investie, pour reprendre, en partie, une citation de Marie Scarpa évoquée plus haut, du "rôle (...) d'explorer " la côte mal taillée en quoi consiste le réel" ", c'est-à-dire l'état 
de l'écriture de cette période de l'Histoire de l'Algérie, encore dévolue à des personnages-historiens "non initiés sur-initiés", des historiens-témoins liminaires, encore vivants mais prisonniers des morts.

\section{BIBLIOGRAPHIE}

ADAM J.-M., GOLDEINSTEIN J.-P., « La production du sens », Linguistique et discours littéraire, théorie et pratique des textes, Larousse Université, Collection L, 1976, pp. 122-131.

BOUDJEDRA R., Le Démantèlement, Denoël, 1982.

DROUET G., « Le bâton de Jean Valjean. Marcher, sculpter, raconter », Poétique, n 140, 2004/4, pp. 467-482.

DUCHET C., « Roman et objets : l'exemple de Madame Bovary », in DUCHET et al., Travail de Flaubert, Seuil, 1983.

POETIQUE $n^{\circ} 140$, Seuil, novembre 2004.

RICARDOU J., Le Nouveau roman, Collection Points Essais, Seuil, 1990.

SCARPA M., L'Eternelle jeune fille. Une ethnocritique du Rêve de Zola, Edition Honoré Champion, 2009 a.

SCARPA M., « Le personnage liminaire », Romantisme, n 145, 2009 /3 b, pp. 25-35.

GAUCHER CH., «L'altérité des sourds : deux lieux communs pour interroger la liminalité des sociétés individualistes ». Disponible sur le site : http://www.mondecommun.com/uploads/PDF/ Gaucher.pdf

WHALEN T., « De la Rhétorique Considérée comme une Praxis de la Liminalité ». Disponible sur le site : http://uregina.ca/ rheaults/rhetor/2004/whalen-f.pdf

\section{NOTES}

1. Nous pouvons citer l'étude que propose Claude Duchet dans son analyse des objets de Madame Bovary : «Roman et objets : l'exemple de Madame Bovary », in Duchet C. et al., Travail de Flaubert, Seuil, 1993, ou celle sur le même texte de Flaubert: "La production du sens », in Jean-Michel Adam, Jean-Pierre Goldenstein, Linguistique et discours littéraire, théorie et pratique des textes, Larousse Université, Collection L, 1976, pp. 122-131.

2. Ricardou, J., Le Nouveau Roman, Collection Points Essais, Seuil, 1990.

3. Article également disponible sur le site : www.cairn.info/revue-poétique-2004-4 page-467.htm.

4. G. Drouet cite A. J. Greimas Dans l'Attente de l'inattendu, de l'imperfection, Périgueux, Fanlac, 1987, p. 91.

5. L'anthropologue Victor Turner a élaboré son concept de la «liminalité » dans son ouvrage intitulé The Ritual Process (1969). Trd. française : Le Phénomène rituel. Structure et contre-structure, Paris, PUF, 1990. 
6. «La liminalité faisait référence aux espaces sociaux marginaux, au-delà (trans) des contraintes de la vie courante, où les acteurs sont affranchis (ils ont trans-gressé, c'est-à-dire franchi le seuil) de la routine habituelle", cité par Whalen T. dans "De la Rhétorique Considérée comme une Praxis de la Liminalité ». Disponible sur le site : http://uregina.ca/ rheaults/rhetor/2004/whalen-f.pdf 7. Cité par Charles Gaucher dans «L'altérité des sourds : deux lieux communs pour interroger la liminalité des sociétés individualistes ». Disponible sur le site : http://www.mondecommun.com/ uploads/PDF/Gaucher.pdf

\section{RÉSUMÉS}

Nous allons, dans le cadre de cette étude, appréhender la textualisation d'un objet auquel le personnage principal du Démantèlement de Rachid Boudjedra, Tahar El Ghomri, tient précieusement et dont il ne se sépare pas pendant plus de vingt ans: une photographie de lui avec ses quatre amis et une dizaine d'autres camarades de combat, au maquis, pendant la guerre d'Algérie. Nous tenterons de démontrer que cette "photographie de guerre » a une triple dimension : embrayeur narratif pour l'introduction de l'histoire de chaque personnage, elle est aussi le double métaphorique du personnage principal qui la détient et celui de l'écriture de l'Histoire de la guerre de libération.

In this study, we will apprehend the textualisation of an object to which the main character of The Dismantling of Rachid Boudjedra, Tahar El Ghomri, is carefully and it separates never for more than twenty years: a photograph of him with his four friends and a dozen other classmates of battle, during the war of Algeria. We will attempt to demonstrate that this 'war photography' has a double dimension: it is, at the same time, narrative catalyst for the introduction of the history of each character, and double metaphoric main character holding and the writing of the History of the war of liberation.

\section{INDEX}

Mots-clés : Ethnocritique, photographie de guerre, objet culturel, écriture de l'Histoire, Rachid Boudjedra

Keywords : Ethnocriticism, War photography, cultural object, writing of history, Rachid Boudjedra

\section{AUTEUR}

\section{MOUNYA BELHOCINE}

Université A. Mira - LAILEMM - Bejaia 